\title{
COVID-19 Attacks the Kidney: Ultrastructural Evidence for the Presence of Virus in the Glomerular Epithelium
}

\author{
Mauro Abbate ${ }^{a}$ Daniela Rottoli ${ }^{a}$ Andrea Gianatti ${ }^{b}$ \\ aDepartment of Molecular Medicine, Istituto di Ricerche Farmacologiche Mario Negri IRCCS, Bergamo, Italy; \\ ${ }^{b}$ Department of Laboratory Medicine, Anatomic Pathology, Azienda Ospedaliera ASST Papa Giovanni XXIII, \\ Bergamo, Italy
}

Upon severe acute respiratory syndrome coronavirus 2 (SARS-CoV-2) infection, individual patients may experience a series of different clinical settings, ranging from an asymptomatic condition to a life-threatening disease possibly amenable to combined and differential drug treatments. In most severe coronavirus disease 2019 (COVID-19) cases, uncontrolled immune response that triggers the massive proliferation of immune cells and the overproduction of cytokines appears to mediate circulatory failure and multi-organ dysfunction. Among the affected organs, the kidney is an emerging target in COVID-19 complications, and abnormal kidney function is a significant risk factor of death in severely ill patients.

According to a prospective cohort study [1], 44\% of COVID-19 patients had proteinuria and 27\% had hematuria at hospital admission, while $5 \%$ of patients experienced acute kidney injury during in-hospital. Patients with kidney disease had a significantly higher risk for death.

Autopsy studies are becoming available that point to the possibility of a direct cytopathic effect of SARSCoV-2 on renal cells $[2,3]$. In early April in Bergamo, a 93-year-old woman was admitted to the hospital with a

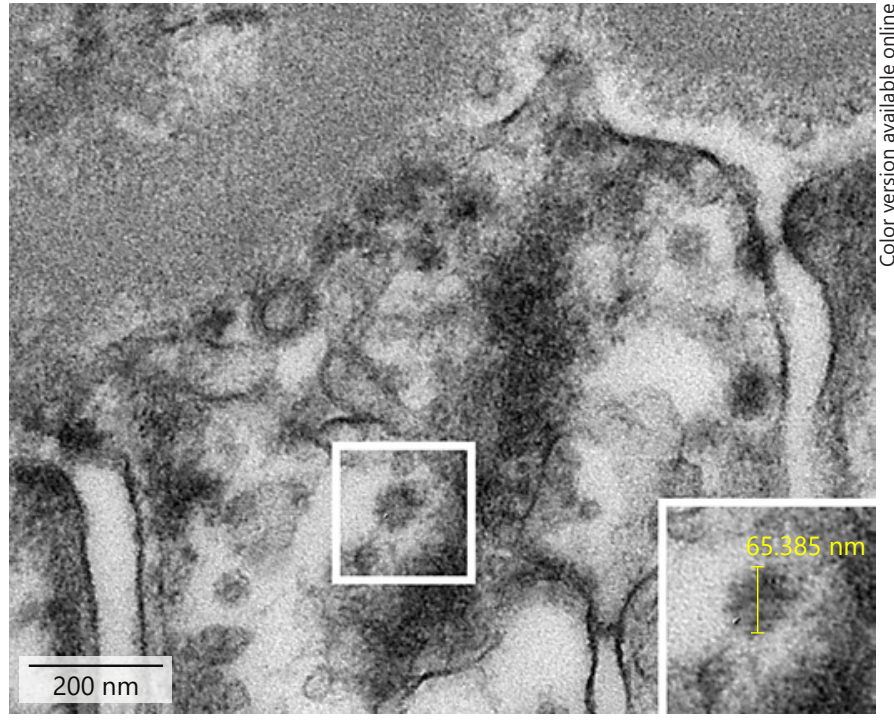

Fig. 1. Transmission electron microscopy image of a foot process of podocyte, anchored to the glomerular basement membrane visible on top, and 2 adjacent portions of foot processes. Morphology of coronavirus with recognizable spikes, enlarged in the inset, and other particles are indicative for the presence of SARS-CoV-2. SARS-CoV-2, severe acute respiratory syndrome coronavirus 2. karger@karger.com www.karger.com/nef

(c) 2020 S. Karger AG, Basel

Karger ${ }^{\prime}=$
Dr. Mauro Abbate

Centro Anna Maria Astori, Science and Technology Park Kilometro Rosso Istituto di Ricerche Farmacologiche Mario Negri IRCCS

Via Stezzano, 87, IT-24126 Bergamo (Italy)

mauro.abbate@ marionegri.it 
traumatic injury. Because of her worsening dyspnea at admission and early respiratory failure, a nasopharyngeal swab was done to test SARS-CoV-2 RNA by RT$\mathrm{PCR}$, which proved positive. The respiratory failure rapidly deteriorated and the patient died 2 days after the trauma. She had a history of hypertension, diabetes, and ESRD. The analysis of kidney tissue by transmission electron microscopy taken 12 -h postmortem reveals viral particles with typical features of coronavirus, indicating SARS-CoV-2 in podocytes (Figure 1). The case of this patient represents the first documented patient, in Europe, with evidence for the presence of SARS-CoV-2 in the kidney. Whether the renal involvement or other concomitant factors had a critical impact on respiratory failure and death is not clear. Very harmful consequences of injured glomerular epithelium in COVID-19 patients can be envisioned particularly in the acute setting and diabetes. They include capillary barrier dysfunction and proteinuria, hematuria, altered coagulation, and worsening of edema among multiple factors. The evaluation of kidney function should be taken into account timely in every patient at risk.

\section{Statement of Ethics}

In Italy, autopsy does not require informed consent from relatives. (Diagnostic assessment, National law 15 February 1961, Decree dated 10 September 1990, n.285. Approval of mortuary police regulations)

\section{Disclosure Statement}

The authors have no conflicts of interest to declare.

\section{Author Contributions}

M.A. designed the study, carried out the electron microscopy analysis and wrote the manuscript. D.R. carried out sample preparation. A.G. collected clinical data and performed the autopsy. All authors have read and approved the final version to be published.

\section{References}

1 Cheng Y, Luo R, Wang K, Zhang M, Wang $Z$, Dong L, et al. Kidney disease is associated with in-hospital death of patients with COVID-19. Kidney Int. 2020;97(5):82938.

2 Su H, Yang M, Wan C, Yi L, Tang F, Zhu H, et al. Renal histopathological analysis of 26 postmortem findings of patients with COVID-19 in China. Kidney Int. 2020.

3 Pan X, Xu D, Zhang H, Zhou W, Wang L, Cui $\mathrm{X}$. Identification of a potential mechanism of acute kidney injury during the COVID-19 outbreak: a study based on single-cell transcriptome analysis. Intensive Care Med. 2020. 